Special issue of the International Conference on Computational and Experimental Science and Engineering (ICCESEN 2014)

\title{
Serret-Frenet Formulae for Null Quaternionic Curves in Semi Euclidean 4-Space $\mathbb{R}_{1}^{4}$
}

\author{
A. Tuna AksoY* And A.C. ÇÖKen
}

Süleyman Demirel University, Department of Mathematics, Isparta, Turkey

Akdeniz University, Department of Mathematics, Antalya, Turkey

\begin{abstract}
In this study, we construct the Cartan frame of a null quaternionic curves in semi Euclidean spaces $R_{1}^{4}$ for an arbitrary parameter.
\end{abstract}

DOI: 10.12693/APhysPolA.128.B-293

PACS: $02.40 .-\mathrm{k}, 02.40 . \mathrm{Hw}$

\section{Introduction}

In a semi-Euclidean spaces, there exist three families of quaternionic curves, that is, spacelike, time-like, and null or lightlike quaternionic curves, according to their causal characters. The theory of Frenet frames for a semi-real quaternionic curve has been studied and developed by several researchers in this field (cf. [1] and [2]). In Bharathi and Nagaraj studied a Frenet frame for a quaternionic curve in $\mathbb{R}^{3}$ and $\mathbb{R}^{4}$, and gave the Frenet formulas for it. Coken and A. Tuna $[2,3]$ studied the differential geometry of a quaternionic curve in a 4dimensional semi-Euclidean space $\mathbb{R}_{2}^{4}$. And then, we study Frenet formulae for null quaternionic curve in $\mathbb{R}_{1}^{3}$ and $\mathbb{R}_{2}^{3}$. But, to our knowledge, there has been no study on the Serret Frenet formulae for null quaternionic curves in $\mathbb{R}_{1}^{4}$.

In this paper we discuss Cartan frame for null quaternionic curves in the 4-dimensional semi Euclidean space $\mathbb{R}_{1}^{4}$. In this section, following [2] and [4], we use the proposition [4] and Cartan Frame for a null quaternionic curve in $\mathbb{R}_{1}^{3}[5]$.

Let $h$ denote the quaternion metric on $\mathbb{R}_{1}^{4}$. A quaternionic curve $\beta(s)$ in $\mathbb{R}_{1}^{4}$ is called a null quaternionic curve if $h\left(\beta^{\prime}(s), \beta^{\prime}(s)\right)=0$ and $\beta^{\prime}(s) \neq 0$ for all $s$. We note that a null curve $\beta(s)$ in $\mathbb{R}_{1}^{4}$ satisfies $h\left(\beta^{\prime \prime}(s), \beta^{\prime \prime}(s)\right) \geq$ 0 (cf. [4, Ch. 1]). We say that a null quaternionic curve $\beta(s)$ in $\mathbb{R}_{1}^{4}$ is parametrized by the pseudo-arc if $h\left(\beta^{\prime \prime}(s), \beta^{\prime \prime}(s)\right)=1,[5]$.

\section{Preliminaries}

Let we give basic concept about the semi-real quaternions. The set of the semi real quaternions

$$
\begin{gathered}
\mathbb{Q}_{\nu}=\left\{q \mid q=a e_{1}+b e_{2}+c e_{3}+d ; a, b, c, d \in \mathbb{R},\right. \\
e_{1}, e_{2}, e_{3} \in \mathbb{R}_{\nu}^{3} ;(\nu=1,2), h_{\nu}\left(e_{i}, e_{i}\right)=\varepsilon\left(e_{i}\right), \\
1 \leq i \leq 3\},
\end{gathered}
$$

*corresponding author; e-mail: abidebytr@yahoo.com where

$$
\begin{aligned}
& e_{i} \times e_{i}=-\varepsilon\left(e_{i}\right), 1 \leq i \leq 3 \\
& e_{i} \times e_{j}=\varepsilon\left(e_{i}\right) \varepsilon\left(e_{j}\right) e_{k} \text { in } \mathbb{R}_{1}^{3} \\
& e_{i} \times e_{j}=-\varepsilon\left(e_{i}\right) \varepsilon\left(e_{j}\right) e_{k} \text { in } \mathbb{R}_{2}^{4}
\end{aligned}
$$

and $(i j k)$ is an even permutation of (123). The multiplication of two semi real quaternions $\mathrm{p}$ and $\mathrm{q}$ is defined by

$$
p \times q=S_{p} S_{q}+S_{p} V_{q}+S_{q} V_{p}+h\left(V_{p}, V_{q}\right)+V_{p} \wedge V_{q}
$$

for every $p, q \in \mathbb{Q}_{\nu}$,

where we have used the inner and cross products in semi-Euclidean space $\mathbb{R}_{\nu}^{3}$. For a semi real quaternion $q=a e_{1}+b e_{2}+c e_{3}+d \in \mathbb{Q}_{\nu}$ the conjugate $\alpha q$ of $q$ is defined by $\alpha q=-a e_{1}-b e_{2}-c e_{3}+d$ Thus, we define symmetric, non-degenerate valued bilinear form $h$ as follows:

$$
h_{\nu}: \mathbb{Q}_{\nu} \times Q_{\nu} \rightarrow R
$$

by

$$
\begin{aligned}
& h_{1}(p, q)=\frac{1}{2}[\varepsilon(p) \varepsilon(\alpha q)(p \times \alpha q)+\varepsilon(q) \varepsilon(\alpha p)(q \times \alpha p)] \\
& \quad \text { for } \mathbb{R}_{1}^{3}
\end{aligned}
$$

and

$$
\begin{aligned}
& h_{2}(p, q)=\frac{1}{2}[-\varepsilon(p) \varepsilon(\alpha q)(p \times \alpha q)-\varepsilon(q) \varepsilon(\alpha p)(q \times \alpha p)] \\
& \quad \text { for } \mathbb{R}_{2}^{4},
\end{aligned}
$$

where

$$
\begin{aligned}
h_{\nu}(q, q) & =a^{2} \varepsilon\left(e_{1}\right)+b^{2} \varepsilon\left(e_{2}\right)+c^{2} \varepsilon\left(e_{3}\right)+d^{2} \\
\quad \text { for } \nu & =\{1,2\}
\end{aligned}
$$

is called the semi-real quaternion inner product. The vector product of two semi real quaternions $p=a_{1} e_{1}+b_{1} e_{2}+$ $c_{1} e_{3}+d_{1}$ and $q=a e_{1}+b e_{2}+c e_{3}+d$ is defined as

$$
\begin{aligned}
V_{p} & \wedge V_{q}=\varepsilon\left(e_{2}\right) \varepsilon\left(e_{3}\right)\left(b_{1} c-b c_{1}\right) e_{1}-\varepsilon\left(e_{1}\right) \varepsilon\left(e_{3}\right) \\
& \times\left(a_{1} c-a c_{1}\right) e_{2}+\varepsilon\left(e_{1}\right) \varepsilon\left(e_{2}\right)\left(a_{1} b-a b_{1}\right) e_{3}
\end{aligned}
$$$$
\text { for } \nu=1
$$

and

$$
\begin{aligned}
& V_{p} \wedge V_{q}=-\varepsilon\left(e_{2}\right) \varepsilon\left(e_{3}\right)\left(b_{1} c-b c_{1}\right) e_{1}+\varepsilon\left(e_{1}\right) \varepsilon\left(e_{3}\right) \\
& \times\left(a_{1} c-a c_{1}\right) e_{2}-\varepsilon\left(e_{1}\right) \varepsilon\left(e_{2}\right)\left(a_{1} b-a b_{1}\right) e_{3} \\
& \quad \text { for } \nu=2 .
\end{aligned}
$$


The norm of semi real quaternion $q$ is denoted by

$$
\|q\|^{2}=\left|h_{\nu}(q, q)\right|=\left|a^{2} \varepsilon\left(e_{1}\right)+b^{2} \varepsilon\left(e_{2}\right)+c^{2} \varepsilon\left(e_{3}\right)+d^{2}\right|
$$

for $\nu=\{1,2\}$.

The concept of a spatial quaternion will be made use throughout our work. $q$ is called a spatial quaternion whenever $q+\alpha q=0$. It is a temporal quaternion whenever $q-\alpha q=0[1-3,5]$.

Theorem I: (Let $e_{1}$ be a time-like vector.)

Let $\mathbb{R}_{1}^{3}$ denote a 3 -dimensional semi-Euclidean space with quaternionic metric $h$. Let $\gamma: I \rightarrow \mathbb{R}_{1}^{3}$ be a null quaternionic Cartan curve such that $\left\{\gamma^{\prime}(s), \gamma^{\prime \prime}(s), \gamma^{\prime \prime \prime}(s)\right\}$ is positively or negatively oriented for all $s \in I$. Let us consider its Cartan frame $\left\{l=\gamma^{\prime}(s), n, u\right\}$ where $h(l, l)=$ $h(n, n)=h(l, u)=h(n, u)=0, h(l, n)=h(u, u)=1$. The Frenet equations are

$$
l^{\prime}=k u, n^{\prime}=\tau u, u^{\prime}=-\tau l-k n
$$

and

$$
l^{\prime}=k u, n^{\prime}=-\tau u, u^{\prime}=\tau l-k n,
$$

where $\tau$ denotes covariant derivative and $k$ is the curvature of the curve. In case $\gamma$ is a null quaternionic Cartan curve, labeling $k(s)=1$, then the Frenet formula of $\gamma(s)$ with respect to $\{l, n, u\}$ becomes [5]

$$
l^{\prime}=u, n^{\prime}=\tau u, u^{\prime}=-\tau l-n
$$

and

$$
l^{\prime}=u, n^{\prime}=-\tau u, u^{\prime}=\tau l-n .
$$

Theorem II: (Let $e_{2}$ be a time-like vector.)

Let $\mathbb{R}_{1}^{3}$ denote a 3 - dimensional semi-Euclidean space with quaternionic metric $h$. Let $\gamma: I \rightarrow \mathbb{R}_{1}^{3}$ be a null quaternionic Cartan curve such that $\left\{\gamma^{\prime}(s), \gamma^{\prime \prime}(s), \gamma^{\prime \prime \prime}(s)\right\}$ is positively or negatively oriented for all $s \in I$. Let us consider its Cartan frame $\left\{l=\gamma^{\prime}(s), n, u\right\}$ where $h(l, l)=h(n, n)=h(l, u)=h(n, u)=0, h(l, n)=$ $-1, h(u, u)=1$. The Frenet equations are

$$
l^{\prime}=k u, n^{\prime}=-\tau u, u^{\prime}=-\tau l+k n
$$

and

$$
l^{\prime}=k u, n^{\prime}=\tau u, u^{\prime}=\tau l+k n,
$$

where $\tau$ denotes covariant derivative and $k$ is the curvature of the curve. In case $\gamma$ is a null quaternionic Cartan curve, labeling $k(s)=1$, then the Frenet formula of $\gamma(\mathrm{s})$ with respect to $\{l, n, u\}$ becomes [5]

$$
l^{\prime}=u n^{\prime}=-\tau u, u^{\prime}=-\tau l+n
$$

and

$$
l^{\prime}=u, n^{\prime}=\tau u, u^{\prime}=\tau l+n .
$$

Now let us begin to set up the Frenet equations of a null quaternionic curve by using the main results of [4, proposition 2.3, page 7] and Theorems I and II.

\section{Serret-Frenet formulae for null quaternionic curves in the semi-Euclidean spaces $\mathbb{R}_{1}^{4}$}

Case I: (Let $e_{1}$ be a time-like vector.)

An ortonormal basis $\left\{e_{1}, e_{2}, e_{3}, e_{4}=1\right\}$ of $\mathbb{R}_{1}^{4}$. Construct vectors

$$
L=\frac{1}{\sqrt{2}}\left(e_{2}+e_{1}\right), N=\frac{1}{\sqrt{2}}\left(e_{2}-e_{1}\right), U=e_{3}, W=1
$$

which satisfy

$$
\begin{aligned}
& h(L, L)=h(N, N)=h(L, U)=h(N, U)=h(W, U)= \\
& h(N, W)=h(L, W)=0
\end{aligned}
$$

and

$$
h(L, N)=h(U, U)=h(W, W)=+1 .
$$

Thus $\{L, N, U, W\}$ is a basis of $\mathbb{R}_{1}^{4}$ which constains $L$ and $N$ null vectors and $U$ and $W$ spacelike vectors. Let $\mathbb{R}_{1}^{4}$ denote a 4-dimensional semi-Euclidean space with quaternionic metric $h$. The 4-dimensional semi-Euclidean spaces in $\mathbb{R}_{1}^{4}$ are identified with the spaces of unit quaternion. Let $\beta: I \subset \mathbb{R} \rightarrow \mathbb{Q}_{\nu}, \beta=\gamma_{1} e_{1}+\gamma_{2} e_{2}+\gamma_{3} e_{3}+\gamma_{4} e_{4}$ be a null quaternionic curve in $\mathbb{R}_{1}^{4}$ defined over the interval I. Let the distinguished parameter $s$ be chosen such that the tangent $L(s)=\sum_{i=1}^{4} \gamma_{i}^{\prime}(s) e_{i}$ has zero length. Let $\{L(s), N(s), U(s), W(s)\}$ be the Frenet apparatus of the differentiable semi-Euclidean space curve in the semiEuclidean spaces $\mathbb{R}_{1}^{4}$. We denote the Frenet frame and Frenet equations of this particular class of $\beta$ by $[4$, proposition 2.3, Case I]. Then Frenet equations are

$$
\begin{aligned}
& L^{\prime}=K W N^{\prime}=-(\tau+p) U+p W \\
& U^{\prime}=(\tau+p) L W^{\prime}=-p L-K N
\end{aligned}
$$

and

$$
\begin{aligned}
& L^{\prime}=K W N^{\prime}=(\tau-p) U+p W \\
& U^{\prime}=-(\tau-p) L W^{\prime}=-p L-K N .
\end{aligned}
$$

Let $\beta$ be a null quaternionic curve, with the tangent $L(s)=\beta^{\prime}(s)=\sum_{i=1}^{4} \gamma_{i}^{\prime}(s) e_{i}$ has zero length. Then we have

$$
h\left(\beta^{\prime}(s), \beta^{\prime}(s)\right)=\beta^{\prime}(s) \times \alpha \beta^{\prime}(s)=0, \beta^{\prime}(s) \neq 0 .
$$

Since $\beta^{\prime}(s)=L$ is a null, we have $h(L, L)=0$. But we do not allow $\beta^{\prime \prime}(s)=W$ to be null, so it is always spacelike. We also have

$$
h(W, W)=h\left(\beta^{\prime \prime}(s), \beta^{\prime \prime}(s)\right)=1 .
$$

Differentiating of equation (3.1) with respect to $s$,

$$
\beta^{\prime \prime}(s) \times \alpha \beta^{\prime}(s)+\beta^{\prime}(s) \times \alpha \beta^{\prime \prime}(s)=0
$$

is obtained. Then, this equation follows that

(i) $\beta^{\prime \prime}(s) \times \alpha \beta^{\prime}(s)$ is a semi spatial quaternion,

(ii) $h\left(\beta^{\prime}(s), \beta^{\prime \prime}(s)\right)=0 \Rightarrow h(L, W)=0$ $h\left(L, L^{\prime}\right)=0$.

If we use the differentiation of (ii), we obtained that $h\left(L W^{\prime}\right)=-1$. Now, $L^{\prime}$ is a vector and can be written in terms of any basis. With the orthonormal basis $\left\{L, N, U, W, L^{\prime}\right.$ becomes,

$$
L^{\prime}=a L+b N+c U+d W, a, b, c, d \in \mathbb{R} .
$$

Taking the scalar product of equation (3.3) with $L, N, U$ and $W$, respectively. Since $L^{\prime}$ itself is a semi-spatial 
quaternion, we define the semi-spatial quaternion $W$ and the scalar function $K$ by means of the following equation

$$
L^{\prime}=K W, h\left(L^{\prime}, W\right)=K=1
$$

If we use the differentiation of $\|L\|=H(L, L)=0$ and (3.4) in the resulting equation, we obtain that

$$
W \times \alpha L+L \times \alpha W=0
$$$$
\text { because of } K(s) \neq 0 \text {. }
$$

Then, this equation follows that

(i) $W$ is $h$-orthogonal to $L, h(L, W)=0$

(ii) Since $l$ is a semi-spatial quaternion, $L \times \alpha W$ is a semi-spatial quaternion.

Then, it is obvious that $l$ is null and $W$ is spacelike unity. Let $L=l \times W$ be null quaternionic vector, along $\beta$, we can now write $L$ as follows

$$
l=L \times \alpha W \text { and } L=l \times W
$$

Using differentiation of Eq. (3.5) and Eqs. (3.4) and (2.1) in the resulting equation, we obtain that

$$
U=u \times W \text { and } l \times W^{\prime}=K W-k U .
$$

The properties of $U$ are,

(i) $h(U, U)=U \times \alpha U=1$

(ii) $L U$ and $W$ are mutually $h$-orthogonal since $l$ and $u$ are so.

By differentiating $U=u \times W$ given by Eq. (3.6), using the Eqs. (2.1) and (3.5) in the resulting equation, we have

$$
U^{\prime}=\tau L-k N+u \times W^{\prime} \text { where } N=n \times W
$$

The properties of $N$ are,

(i) $|N|^{2}=h(N, N)=0$

(ii) $N, U$ and $W$ are mutually $h$-orthogonal since $n$ is so. Then $L$ and $N$ are mutually $h$-orthogonal since $h(L, N)=1$ is so.

By differentiating $N=n \times W$ given by Eq. (3.7), using the Eqs. (2.1) and (3.6) in the resulting equation, we have

$$
N^{\prime}=-\tau U+n \times W^{\prime}
$$

Consequently, we get

$$
\begin{aligned}
& l \times W^{\prime}=K W-k U, \quad U=u \times W \\
& U^{\prime}=\tau L-k N+u \times W^{\prime}, \quad N=n \times W, \\
& N^{\prime}=-\tau U+n \times W^{\prime} .
\end{aligned}
$$

With the way viewed up here, among the Frenet equataions, just the (3.4) equation has been obtained. In order to get Frenet equations by the method below;

$$
\begin{aligned}
& L^{\prime}=a_{11} L+a_{12} N+a_{13} U+a_{14} W \\
& N^{\prime}=a_{21} L+a_{22} N+a_{23} U+a_{24} W \\
& U^{\prime}=a_{31} L+a_{32} N+a_{33} U+a_{34} W \\
& W^{\prime}=a_{41} L+a_{42} N+a_{43} U+a_{44} W
\end{aligned}
$$

Taking the scalar product of these equations with $L, N, U$ and $W$, respectively, we find that

$$
\left[\begin{array}{c}
\mathrm{L}^{\prime} \\
\mathrm{N}^{\prime} \\
\mathrm{U}^{\prime} \\
\mathrm{W}^{\prime}
\end{array}\right]=\left[\begin{array}{cccc}
0 & 0 & 0 & K \\
0 & 0 & m & p \\
-m & 0 & 0 & r \\
-p & -K & -r & 0
\end{array}\right]\left[\begin{array}{c}
L \\
N \\
U \\
W
\end{array}\right]
$$

We obtain $W^{\prime}$ from this matrix as follows:

$$
W^{\prime}=-p L-K N-r U
$$

and if we have the quaternion multiplication of $W^{\prime}$ vector by $u, n$ and $l$ vectors, we get

$$
\begin{aligned}
& l \times W^{\prime}=K W+K U-r L, \\
& n \times W^{\prime}=p W-p U+r N, \\
& u \times W^{\prime}=p L-K N+r W .
\end{aligned}
$$

Hence, substituting Eq. (3.11) in the Eq. (3.9), we obtain that

$$
\begin{aligned}
& (K+k) U+r L=0, \\
& N^{\prime}=(-\tau-p) U+p W+r N, \\
& U^{\prime}=(\tau+p) L-(K+k) N+r W .
\end{aligned}
$$

Hence we have equation (3.13) of the following form

$$
\left[\begin{array}{c}
\mathrm{L}^{\prime} \\
\mathrm{N}^{\prime} \\
\mathrm{U}^{\prime} \\
\mathrm{W}^{\prime}
\end{array}\right]=\left[\begin{array}{cccc}
0 & 0 & 0 & K \\
0 & 0 & -\tau-\mathrm{p} & p \\
\tau+p & -(K+k) & 0 & r \\
-p & -K & -r & 0
\end{array}\right]\left[\begin{array}{c}
L \\
N \\
U \\
W
\end{array}\right]
$$

If the matrices in Eqs. (3.13) and (3.10) are equaled, we have $r=0$ because $K+k=0$. Consequently, we get

$$
\left[\begin{array}{c}
\mathrm{L}^{\prime} \\
\mathrm{N}^{\prime} \\
\mathrm{U}^{\prime} \\
\mathrm{W}^{\prime}
\end{array}\right]=\left[\begin{array}{cccc}
0 & 0 & 0 & K \\
0 & 0 & -\tau-\mathrm{p} & p \\
\tau+p & 0 & 0 & 0 \\
-p & -K & 0 & 0
\end{array}\right]\left[\begin{array}{c}
L \\
N \\
U \\
W
\end{array}\right]
$$

By the same method using Eq. (2.2) in $\mathbb{R}_{1}^{3}$ and $K+k=$ 0 in $\mathbb{R}_{1}^{4}$, we get the matrix as follows;

$$
\left[\begin{array}{c}
\mathrm{L}^{\prime} \\
\mathrm{N}^{\prime} \\
\mathrm{U}^{\prime} \\
\mathrm{W}^{\prime}
\end{array}\right]=\left[\begin{array}{cccc}
0 & 0 & 0 & K \\
0 & 0 & \tau-\mathrm{p} & p \\
-(\tau-p) & 0 & 0 & 0 \\
-p & -K & 0 & 0
\end{array}\right]\left[\begin{array}{c}
L \\
N \\
U \\
W
\end{array}\right]
$$

Here, $K$ is the first curvature of $\beta$ curve in $\mathbb{R}_{1}^{4}, k$ is both the first curvature of $\gamma$ curve in $\mathbb{R}_{1}^{3}$ and the opposite signed second curvature of $\beta$ curve in $\mathbb{R}_{1}^{4}$. However, this is not possible. Because, $K=1$ and $k=1$. Whereas from $K+k=0$, it should have been $K=-k$. Thus, when we label $K=1$ and $k=1$, we can not prove the Frenet equations. While $e_{1}$ is time-like in $\mathbb{R}_{1}^{4}$, we do not construct Frenet frame for a null quaternionic curve in $\mathbb{R}_{1}^{4}$.

Case II: (Let $e_{2}$ be a time-like vector.)

An ortonormal basis $\left\{e_{1}, e_{2}, e_{3}, e_{4}=1\right\}$ of $\mathbb{R}_{1}^{4}$ Let $e_{2}$ be a time-like vector. Construct vectors

$$
L=\frac{1}{\sqrt{2}}\left(e_{2}+e_{1}\right), N=\frac{1}{\sqrt{2}}\left(e_{2}-e_{1}\right), U=e_{3}, W=1
$$

which satisfy 


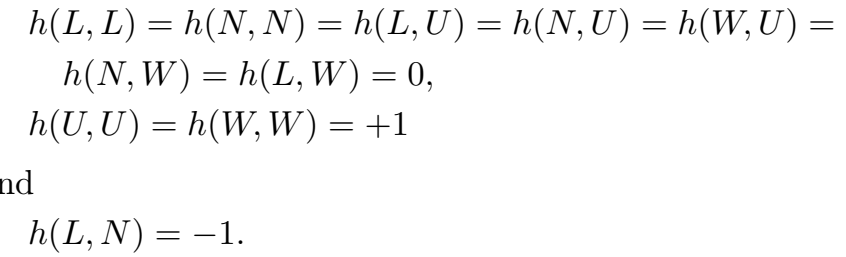

Thus $\{L, N, U, W\}$ is a basis of $\mathbb{R}_{1}^{4}$ which constains $L$ and $N$ null vectors and $U$ and $W$ spacelike vectors. Let $\mathbb{R}_{1}^{4}$ denote a 4-dimensional semi-Euclidean space with quaternionic metric $h$. The four-dimensional semi-Euclidean spaces in $\mathbb{R}_{1}^{4}$ are identified with the spaces of unit quaternion. Let $\beta: I \subset R \rightarrow \mathbb{Q}_{\nu} \quad \beta=\gamma_{1} e_{1}+\gamma_{2} e_{2}+\gamma_{3} e_{3}+\gamma_{4} e_{4}$ be a null quaternionic curve in $\mathbb{R}_{1}^{4}$ defined over the interval $I$. Let the distinguished parameter $s$ be chosen such that the tangent tangent $L(s)=\beta^{\prime}(s)=\sum_{i=1}^{4} \gamma_{i}^{\prime}(s) e_{i}$ has zero length. Let $\{L(s), N(s), U(s), W(s)\}$ be the Frenet apparatus of the differentiable semi-Euclidean space curve in the semi-Euclidean spaces $\mathbb{R}_{1}^{4}$. We denote the Frenet frame and Frenet equations of this particular class of $\beta$ by proposition (Case I). Then Frenet equations are

$$
\begin{aligned}
& L^{\prime}=K W, N^{\prime}=(\tau+p) U+p W, \\
& U^{\prime}=(\tau+p) L, W^{\prime}=p L+K N
\end{aligned}
$$

and

$$
\begin{aligned}
& L^{\prime}=K W, N^{\prime}=-(\tau-p) U+p W \\
& U^{\prime}=-(\tau-p) L, W^{\prime}=p L+K N .
\end{aligned}
$$

Here, $K$ is the first curvature of $\beta$ curve in $\mathbb{R}_{1}^{4}, k$ is both the first curvature of $\gamma$ curve in $\mathbb{R}_{1}^{3}$ and second curvature of $\beta$ curve in $\mathbb{R}_{1}^{4}$. We find that $K=1$ and $k=1$ truly and these values provide $(K-k)=0$. Using the method of theorem 3.2 [2], while $e_{2}$ is time-like in $\mathbb{R}_{1}^{4}$, we construct Frenet frame for a null quaternionic curve in $\mathbb{R}_{1}^{4}$.

\section{Conclusions}

While $e_{1}$ is time-like in $\mathbb{R}_{1}^{4}$, we do not construct Frenet frame for a null quaternionic curve in $\mathbb{R}_{1}^{4}$. While $e_{2}$ is time-like in $\mathbb{R}_{1}^{4}$, we construct Frenet frame for a null quaternionic curve in $\mathbb{R}_{1}^{4}$

\section{Acknowledgments}

This work was supported by the SDU Funding Agency under Grant 2059-D-09.

\section{References}

[1] K. Bharathi, M. Nagaraj, Indian J. Pure Ap. Mat. 18, 507 (1987).

[2] A.C. Cöken, A. Tuna, Appl. Math. Comput. 155 , 373 (2004).

[3] A. Tuna, M.Sc. Thesis, Süleyman Demirel University, Graduate School of Natural and Applied Science, Isparta, Turkey 2002.

[4] L.K. Duggal, A. Bejancu, Lightlike submanifolds of semi-Riemannian manifolds and applications, Kluwer Academics Publishers, Dordrecht 1996, p. 300.

[5] A. Tuna Aksoy, Ph.D. Thesis, Süleyman Demirel University Graduate School of Natural and Applied Science, Isparta, Turkey 2014. 